\title{
Prospective Research Challenges in Wireless Sensor Networks
}

\author{
Hathal Salamah A. Alwageed \\ Department of Computer Engineering and Network \\ Aljouf University, Saudi Arabia
}

\begin{abstract}
Dynamic starting late there has been a creating energy for Wireless Sensor Networks (WSN). Late movements in the field of sensing, enrolling and exchanges have pulled in examination attempts and colossal endeavors from distinctive quarters in the field of WSN. Also such sensing networks will reveal as of now in mystery phenomena. The diverse areas where noteworthy examination activities proceeding in the field of WSN are exploitation, localization, synchronization, data gathering/aggregation, dissemination, database addressing or querying, structural design, middleware, security, sketching out less power exhausting contraptions (devices), and towering algorithms for specific dilemmas of WSN's. Here we give an outline of constant investigation works out, distinctive arrangement issues included and possible game plans melding these issues.
\end{abstract}

\section{Keywords}

WSN's, Communication, Sensor networks, problems

\section{INTRODUCTION}

Adaptable correspondence/communications such as Mobile communication and technology of wireless networking development have seen a thriving change recently. Driven by imaginative types of progress furthermore application demands diverse classes of correspondence/communication Networks or communication networks have created, for instance, Cellular Networks/networks, Ad hoc Networks, Sensor Networks and Mesh Networks.

Cell Networks are the establishment or infrastructure ward networks. Ad-hoc Networks are portrayed as the arrangement of remote/wireless Networks that make use of multi-hop radio relaying after the Ad-hoc network are quickly and selfdecisively found. Ad-hoc Networks are without system Networks [1]. As showed by Akylidiz et al., WSN comprises extensive number of Ad-hoc network that are sent in such a way, to the point that they can sense the phenomena [2]. Akkaya and Younis describe WSN as a network that includes minute node with distinguishing, retribution and correspondence/communication limits [3]. In spite of the way that sensor Networks are an extraordinary kind of Ad-hoc Networks, the protocols planned for Ad-hoc Networks can't be used as it is for sensor orchestrates as a result of the going hand in hand with reasons. The number of Ad-hoc network in sensor Networks is broad and needs relative to a couple of solicitations of degree more than the Ad-hoc networks and thus requires various and more flexible game plans. The data rate is depended upon to be low in WSN and is of true in nature. Regardless, adaptable Ad-hoc network (MANET) is expected to pass on rich intuitive media data and is mostly sent for passed on transforming. A sensor network is typically passed on by a lone proprietor however MANET is by and large run by a couple of unessential substances. [4] Sensor Networks are data driven i.e. the request in sensor network/network are had a tendency to Ad-hoc network which have data satisfying a couple of conditions and fascinating having a tendency to is unfeasible as they don't have overall identifiers. In any case, MANET is node point driven, with inquiries had a tendency to particular Ad-hoc network dictated by their uncommon areas. Sensor nodes are normally sent once in their life time and those Ad-hoc networks are generally stationary except for two or three convenient Adhoc network, while nodes in MANET move in an extraordinarily selected manner. Like MANET sensor nodes are also expected for self game plan, yet the refinement in movement/traffic and energy usage oblige separate courses of action. In relationship to extraordinarily delegated Networks, sensor nodes have compelled power supply and resuscitate of power is impossible considering the extensive number of Adhoc network and the earth in which they are passed on. Thusly energy usage in WSN is a basic metric to be considered. Sensor Networks are application specific. One can't have an answer that fits for all the issues. Simplicity is the rule in the WSN. Since sensor Ad-hoc networks are squat and there is control on energy usage; the passing on and computing programming or software's in the nodes should be of less size and retribution capable than the ordinary programming/software used for the same reason. Sensor Networks have been projected for a mixture of utilization [2][5][6] like Intrusion detection and taking after for military reason, environment monitoring, development acknowledgment for appreciation seismic tremor outlines and to suspect theft, wellbeing application by watching the drug coordinated to the patients and for movement examination.

\section{MISCELLANEOUS DILEMMAS OF WSN'S}

The main harms that impact the network and execution of a WSN are according to the accompanying:

\subsection{The problem of Hardware and Operation System}

WSN's are made out of countless small devices named as node. A sensor node is much of the time consolidated as a node. A Sensor is a device which resources the information and passes the same on to a bit. Sensors are used to evaluate the movements to physical environment like weight, moisture, sound, vibration and changes to the quality of individual like circulatory strain, tension and heartbeat. A Mote involves processor, memory, battery, A/D converter for interfacing with a sensor and a radio transmitter for confining an ad-hoc network. A Mote and Sensor together edge a Sensor Node. There can be assorted Sensors for unmistakable purposes mounted on a Mote. Bits are also as a less than dependable 
rule suggested as Smart Dust. A Sensor Node shapes a principal unit of the sensor network $[14,15]$. The Ad-hoc network used as a piece of sensor Networks are little and have discriminating energy confinements. The gear design issues of sensor Ad-hoc network are not exactly the same as diverse applications and they are [16]: Radio Range of Ad-hoc network should be high 1-5 kilometers. Radio range is essential for ensuring network combination and data assembling in a network as nature being watched may not have a presented establishment for correspondence/communication. In various Networks the Adhoc network may not make relationship for quite a while or may go out of degree in the wake of constructing connection. Use of Memory Chips like flash memory is recommended for sensor networks as they are non-unusual, sensible and flimsy. Energy/Power Consumption of the recognizing/sensing device should be minimized and sensor nodes should be energy viable since their obliged energy/energy resource chooses their lifetime. To direct power the node point should stop the radio power supply when not being utilized. Battery sort is vital since it can impact the design of sensor nodes. Battery Protection Circuit to evade trick or discharge issue can be added to the sensor nodes. Sensor Networks embodies an immense number of Ad-hoc network. It is supported just if the node is terrible. There are diverse stages that are created considering the above analyzed blueprint issues like Mica2, MicaZ, Telos, BT Node and Imotes and MIT $\mu$ AMPS $(\mu-$ Adaptive Multi-region Power-careful Sensors) [22][23]. Among them the Berkeley Motes, which is fiscally made open by Crossbow Technologies is all that much pervasive and is brought into play by diverse examination affiliations. Berkeley Motes embodies an embedded microcontroller, lowpower radio, streak memory and they are powered by two AA batteries. MICA and MICA2 are the best gatherings of Berkeley bits. The MICA2 stage is outfitted with an Atmel ATmega128L and has a CC1000 handset. A 51-pin improvement connector is open to interface sensors. Microcontrollers are utilized to handle medium access and baseband get ready. An event driven steady living up to expectations system like TinyOS has been executed to unequivocally address the concurrency and resource organization requirements of sensor Ad-hoc network [18][19]. The examinations issues that can be considered are differing systems to upgrade signal reception, design of low power, economical sensors and processing units. Distinctive arrangements to safeguard node point power use and node point headway and essential modulation arrangements may similarly be considered for sensor nodes. An O.S (operation system) framework for a sensor node point should have the ability to give memory and resource management in an obliged circumstance. The distinctive issues in arranging an Operating System (OS) for sensor Networks are: In sensor orchestrate a sensor node point is basically responsible for computation of the removed data from the area environment. It shapes the divided data and controls the data as per the need of an application. Each one of these activities oblige progressing response, get ready and guiding of the data. So concurrency organization is needed in sensor Ad-hoc network. An OS for sensor Ad-hoc network should be hardware independent and application specific. It should support multihop coordinating and clear customer level Networks organization reflections. The OS should have inbuilt highlights to decrease the usage of battery energy. Bits can't be empowered as and when wished on account of minimal size and straightforwardness essential of bits and it should be in a position to actualize limitation on the measure of benefits used by every application [13]. The OS should be need based and it should give need for higher need events. The OS should have a basic programming perfect model. Application originators should have the ability to concentrate on their application method of reasoning instead of being concerned with the low level hardware issues like arranging, appropriating and arranging. Diverse Operating Systems for Sensor Ad-hoc network like TinyOS, Mantis Operating System [9] and Nano-Qplus [10] have been made recollecting the above setup issues. TinyOS is an open source and far by the most pervasive OS balanced by both the examiners and industry alike for introduced sensor Networks. It has been ported on to various stages and sensor devices. According to the information open on the TinyOS site [7] TinyOS has a portion based auxiliary designing enabling quick advancement and utilization while minimizing the code appraise as required by the genuine memory prerequisites which is characteristic in sensor Networks [8]. TinyOS's fragment library fuses network protocols, distributed services, sensor drivers and data securing instruments. The execution model of TinyOS sponsorships complex yet safe concurrent operations. TinyOS has been executed in NesC vernacular [12], which supports the TinyOS part and concurrency model.

\subsection{The Uniqueness of Wireless Radio Communication}

Execution of WSN's depends on upon the nature of remote correspondence/communication (wireless Communication). Nevertheless, remote correspondence/communication (wireless Communication) in sensor Networks is known for its sporadic nature. Rule devising concerns for correspondence/communication in WSNs are: Low power usage in sensor Networks is required to engage long meeting expectations lifetime by promising low commitment cycle operation, low signal processing. Distributed Sensing enough acts against diverse regular obstacles and thought should be taken that the sign quality, thus the feasible radio degree is not lessened by distinctive components like reflection, diffusing and scatterings. Multi-hop Networking may be balanced among sensor nodes to reduce correspondence/communication association/link or connectivity extend besides thickness of sensor Ad-hoc network should be high. Long region correspondence/communication is frequently show point and obliges high transmission power, with the risk of being spied. So short range transmission has been considered to minimize the probability of being spied. Communication systems should fuse error control subsystems to distinguish slips and to right them. Research areas join delineating low power utilizing correspondence/communication systems and complementary metal oxide semiconductor (CMOS) circuit methodology especially streamlined for sensor Networks, arranging new basic building for fused remote sensor structures and change method and data rate decision.

\subsection{Medium Access Approaches}

Correspondence/communication is a genuine wellspring of energy use in WSNs and MAC protocols particularly control the radio of the nodes in the network. Mac protocols should be planned for controlling energy usage, which consequently affects the lifetime of the network [24]. The diverse network issues of the MAC protocols suitable for sensor network environment are: [25] [26] [27] [28] [29]. The MAC layer 
gives fine-grained control of the handset and allows on and off trading of the radio. The diagram of the MAC protocol should have this changing part to pick when and how in many cases the on and off network should be done. This lends a hand in protecting energy.

A MAC protocol should avoid sways from interfering Ad-hoc network, over emitting, getting, control distribute and unmoving tuning in. Exactly when a beneficiary node gets more than one packet meanwhile, these packets are called "affected or collided packets", which need to be sent again in this way growing energy use. Exactly when a destination node point is not arranged to get messages then it is called overemitting. Overhearing happens if a node snatches packet that were headed for some other node point. Sending and tolerating of less important packets realizes control overhead. Unmoving listening is an indispensable component as the Adhoc network every now and again hears the channel for possible reception of the data which is not sent. Scalability, Adaptability and decentralization is another basic measure in laying out a MAC protocol. The sensor network should change in accordance with the changes in the network size, node point thickness and topology. Also a couple of nodes may fail hard additional minutes, some may join and a couple of Ad-hoc network may move to particular territories. A conventional MAC protocol should oblige these movements to the network. A MAC protocol should have minimum latency and high throughput when the sensor Networks are passed on in essential applications. A MAC protocol should join Message Passing. Message passing means differentiating a long message into little parts and transmit them in impact. In this way, a node point which has more data gets of an opportunity time to get to the medium. There should be consistency in reporting the events by a MAC protocol. Since the nodes are sent discretionarily, Ad-hoc network from outstandingly thick district may stand up to high dissension among themselves when reporting events realizing high packet adversity. Consequently the sink recognizes less events from such ranges. Also the Ad-hoc networks which are closer to the sink transmit more packets to the detriment of nodes which are a long way from the sink. The MAC protocols should manage the definitely comprehend issue of Information Asymmetry, which develops if a node is not aware of packet transmissions two hops away. MAC Protocols should satisfy the Real-time requirements. Mac being the base of the correspondence/communication stacks; perfect area, planning and transport of the information from the passed on environment is an essential in a WSN application. Some standard MAC Protocols are S-Mac (Sensor MAC), B-Mac, ZMAC, Time-MAC and WiseMac. Directing energy at MAC layer is a basic examination region by reducing the potential energy wastes which are discussed in the past portion. Some piece of examination must be done tweaking the radio parameters which is the basic wellspring of energy use. Mac protocols should be weaker to the improvement of nodes. The investigation bunch generally neglects flexibility at the MAC layer because sensor Networks were at first anticipated that would exchange off of static Adhoc network. Yet, late works like RoboMote [31] have engaged mobility in sensor Ad-hoc network and there is much space for examination here. Improvement criteria, for instance, absence of movement, pleasantness with steady restrictions or strong data transport for MAC protocols have not grabbed centrality in investigation. The cross layer blueprints in sensor Networks have lead to strong, vertically facilitated game plans which may work for an examination gather however may not be useful for other investigation groups. So making standard sensor network building outline is a constant methodology.

\subsection{Localization}

Sensor constrainment or localization is a vital and basic issue for network management and operation. In a heavy partition of this present reality circumstances, the sensors are passed on without knowing their positions early moreover there is no supporting system available to discover and manage them once they are sent [44][47][48]. Choosing the physical territory of the sensors after they have been passed on is known as the issue of confinement. Region disclosure or constraint estimation for a sensor network should satisfy the going hand in hand with necessities [45]: The confinement count should be flowed since a brought together philosophy obliges high transforming at specific nodes to gage the position of Ad-hoc network in the whole environment. This extends hailing information exchange limit moreover puts extra load on nodes close to node. Knowledge of the node territory can be used to execute energy capable message controlling protocols in sensor Networks. Localization counts should be adequately solid to confine the failure and loss of nodes. It should be tolerant to slip by in physical estimations. It is shown in [46] that the precision of the confinement increases with the amount of signs. A reference point is a node which is aware of its range. Yet, the essential issue with extended signs is that they are more immoderate than other sensor nodes and once the dark stationary Ad-hoc network have been constrained using sign nodes then the aides get the chance to be purposeless. Techniques that depend on after measuring the going information from sign quality and time of passage oblige specific hardware that is frequently not available on sensor nodes. Localization count should be correct, adaptable and reinforce convenience of nodes. The examination on flexible nodes imprisonment and development examination constantly will continue growing as sensor Networks are passed on in significant numbers and as applications get the opportunity to be changed. Scientists in different controls are involved with schedules for taking after the advancements and people incorporate of animals their surroundings i.e. dormant living space checking. Another basic application is to network a system to track the zone of gainful assets in an indoor circumstance. We need to improve the most great likelihood estimation in a scattered area like sensor Networks. Making adaptable helped restriction is another vital examination region. One needs to upgrade the confinement exactness which depends on upon ToA or TDoA.

\subsection{Exploitation}

It refers for setting up an operational sensor sort out in a certifiable circumstance [41]. Association of sensor network is a work concentrated and awkward development as we don't have affect over the way of remote correspondence/communication (wireless Communication) moreover this present reality puts strains on sensor Ad-hoc network by interfering in the midst of exchanges. Sensor Adhoc network can be passed on either by placing reliably in a sensor field or by dropping it from a plane. Diverse sending issues which need to be taken thought are [42] [43]: When sensor Ad-hoc network are passed on in certifiable, Node death on account of energy depletion either made by ordinary battery discharge or due to shortcircuits is a regular issue which may incite wrong sensor readings. In like manner sink 
nodes go about as entries and they store and forward the data assembled. Therefore, issues impacting sink nodes should be perceived to minimize data hardship. Deployment of sensor Networks realizes network blockage due to various synchronous transmission attempts made by a couple sensor nodes. Synchronous transmission attempts happen in view of inappropriate design of the MAC layer or by repeated network surges. Another issue is the physical length of an association. Two Ad-hoc networks may be close to each other yet they will no doubt be not able to pass on as a result of physical impedance in this present reality while nodes which are far away may talk with each other. Low data yield is another typical issue in certifiable association of sensor Ad-hoc network. Low data yield infers a network passes on deficient measure of information. Self Configuration of sensor Networks without human intervention is obliged in light of unpredictable association of sensor Ad-hoc network. A structure is anticipated in [43] considering the above association issues. Power is an item space for orchestrating and passing on remote sensor network applications into real environment. Investigation issues fuse improving the degree and detectable quality of the radio receiving wires when passed on in distinctive physical miracle, perceiving mistakenly sensor readings at the most reliable, to decline lethargy and reduce stopping up.

\subsection{Harmonization/Synchronization}

The service of clock synchronization is a discriminating aspect in sensor Networks. Time Synchronization in sensor network arrangements to give a commonplace timescale to local clocks of Ad-hoc network in the network. An overall clock in a sensor structure will help plan and dismember the data successfully and expect future system conduct. A couple of uses that oblige overall clock synchronization are environment checking, course bearing, vehicle taking after et cetera. A clock synchronization organization for a sensor network needs to meet challenges that are liberally exceptional in connection to those in base based Networks [49][50][51]. Energy usage in some synchronization arrangements is all the more due to energy hungry sorts of apparatus like GPS (Global Positioning System) recipients or NTP (Network Time Protocol). The lifetime or the term for the nodes which are spread over a broad area region needs to be taken into account. Sensor nodes have larger amount of disillusionments. In this way the synchronization protocol needs to be more incredible to disillusionments and to correspondence/communication delay. Sensor nodes need to compose and collaborate to perform an erratic distinguishing undertaking like data blend. In data blend the data accumulated from assorted Ad-hoc network are collected into a huge result. If the sensor nodes need synchronization among themselves then the data estimation will be off course. Traditional synchronization protocols endeavor to finish the most bewildering level of exactness. The higher the accuracy, then there will be more essential for resources. Thusly we need to have trade off between synchronization precision and resource essentials in perspective of the application. Sensor Networks compass multi hops with higher jitter. Thusly, the computation for sensor network clock synchronization needs to accomplish multihop synchronization even in the region of high jitter.

Different synchronization protocols which can be found in the writing are Reference Broadcast Synchronization (RBS) and Delay Measurement Time Synchronization convention.
Different examination issues incorporate building systematic model for multihop synchronization, enhancing the radio correspondence/communication in the current synchronization protocols like RBS (Reference Broadcast Synchronization) and LTS (LightWeight Tree Based Synchronization).

\subsection{Calibration}

It is the strategy of changing the rough sensor readings got from the sensors into cured values by differentiating it and some standard qualities. Manual conformity of sensors in a sensor network is a period eating up and troublesome undertaking due to dissatisfaction of sensor Ad-hoc network and discretionary noise which makes manual arrangement of sensors exorbitantly luxurious. Diverse Calibration issues in sensor Networks are [52][53][54]:

- A sensor network includes tremendous number of sensors frequently with no arrangement interface.

- Access to individual sensors in the field can be confined.

- Reference qualities may not be speedily available.

- Different applications have need of unmistakable arrangement.

- Requires alteration in a brain boggling component environment with various observables like developing, spoiling, damage et cetera.

- Other destinations of arrangement consolidate exactness, quality against discretionary slips, ability to be associated in distinctive circumstances and to address a blended pack of botch models.

Investigation consolidates laying out distinctive modification Networks including the diverse issues which we have discussed heretofore.

\subsection{Problems of Network Layer}

Throughout late years sensor Networks are being made for specific applications and guiding is fundamental for sending the data from sensor Ad-hoc network to Base Station (BS). As discussed, coordinating in sensor Networks is an amazingly troublesome issue. Distinctive issues at the network layer are [36][3][37][39]: Energy viability is a basic standard. We need to discover particular routines to shed energy inefficiencies that may shorten the lifetime of the network. At the network layer, we need to find distinctive Networks for discovering energy capable courses and for giving off the data from the sensor nodes to the BS so that the lifetime of a network can be updated. Routing Protocols should wire multi-way diagram methodology. Multi-way is suggested those protocols which set up different ways so that a route among them can be used when the fundamental way falls level. Path repair is fancied in guiding protocols at whatever point a way break is distinguished. Adjustment to non-discriminating disappointment is another appealing property for guiding protocols. Controlling protocols should have the ability to find another route at the network layer paying little mind to the way that a couple of nodes miss the mark or frustrated on account of some characteristic impedance. Sensor Networks accumulate information from the physical environment and are exceedingly data driven. In the network layer to enlarge energy stores we need to give a versatile stage to performing guiding and data organization. The data development that is 
made will have discriminating abundance among individual sensor Ad-hoc network subsequent to various sensors may create same data inside the locale of a sensation. The directing protocol should attempt such redundancy to upgrade energy and information transmission utilization.

As the nodes are scattered subjectively realizing an offhand coordinating establishment, a directing protocol should have the property of diverse remote skips. Routing Protocols should manage heterogeneous nature of the nodes i.e. each node point will be assorted in wording of retribution, correspondence/communication and power. Diverse sort of routing Protocols for wireless sensor networks are Sensor Protocols for Information via negotiation (SPIN), Rumor Routing, Direct Diffusion, Low Energy Adaptive Cluster Hierarchy (LEACH), Threshold sensitive Energy Efficient sensor Network protocol (TEEN), Geographic and Energy Aware Routing (GEAR), Sequential Assignment Routing (SAR) etc. Sensor Networks are still at an early stage similarly as development as it is still not for the most part sent in genuine and this opens various portals for investigation. The present controlling protocols need to be upgraded as they have their own specific game plan of issues. Much work is not expounded on clash issues or high network development. No resourceful work is completed or carried out.

\subsection{Problems of Transport Layer}

The concern end-to-end strong correspondence/communication has been bestowed at Transport layer. The diverse setup issues for Transport layer protocols are [32][33]:

In vehicle layer the messages are isolated into a couple of bits at the transmitter and reassembled at the beneficiary. Therefore this protocol should certify conscious transmission of the separated parts. Limited bandwidth brings about obstructing which influences conventional data exchange and may moreover provoke packet disaster. Bit mix-up rate similarly achieves group adversity moreover wastes energy. A transport protocol should be trustworthy for passing on data to possibly sweeping get-together of sensors under stunning conditions. End to End correspondence/communication may continue due to diverse reasons: The position of nodes is not predestined and external impediments may realize poor correspondence/communication execution between two Adhoc networks. If this sort of issue is experienced then end to end correspondence/communication will persevere. Another issue is disillusionment of Ad-hoc network due to battery fatigue. In sensor sorts out the loss of data, when it spills out of source to sink is generally fair. Yet, the data that spills out of sink to source is fragile to message adversity. A sensor secures information from the including environment and passes it on to the sink which in this way requests the sensor node point for information. Standard transport protocols, for instance, UDP and TCP can't be particularly executed in sensor Networks for the reason that is, if a sensor node is a long way from the sink then the stream and stopping up control instrument can't be ordered those nodes.

Successful end to end transmissions of packets are guaranteed in TCP yet its excess in an event driven uses of sensor Networks. Overhead in a TCP connection does not work commendably for an event driven utilization of sensor Networks. UDP on the other hand has a reputation of not giving strong data movement and has no stopping up or stream control instruments which are needed for sensor
Networks. [34] Proposed one of the standard transport layer protocol. Making transport protocols for sensor Networks is itself a troublesome errand as a result of the previously inspected issues and almost no work is represented. Existing transport layer protocols for WSNs expect that the network layer uses a lone way coordinating and multi-Path routing is not well thought-out; which opens various gateways for investigation in this heading. Countless vehicle protocols don't consider need when directing. Since sensor Ad-hoc network are placed in distinctive sorts of environment, the data from various ranges will have particular needs.

\subsection{The Aggregation and Dissemination of Data}

Data get-together is the essential objective of sensor nodes. The sensors once in a while sense the data from the enveloping environment, process it and transmit it to the base station or sink. The repeat of reporting the data and the amount of sensors which report the data depends on upon the particular application. Data gathering incorporates purposely assembling the distinguished data from various sensors and transmitting the data to the base station for further planning. In the meantime, the data made from sensors is habitually dull besides the measure of data created may be to a great degree goliath for the base station to process it.

Consequently we oblige a network for solidifying the recognized data into bewildering information and this is attained through Data Aggregation [58]. Data Aggregation is described as the procedure of amassing the data from different sensors to slaughter dull transmission and surveying the needed answer about the distinguished environment, then giving interweaved information to the base station. Some arrangement issues in data aggregate are [58][59]: Sensor Networks are naturally flawed and certain information may be out of reach or luxurious to get; like the amount of nodes present in the network and the amount of Ad-hoc network that are responding besides it is difficult to gain complete and in the current style information of the neighboring sensor Adhoc network to aggregate information. Making a rate of the nodes to transmit the data particularly to the base station or to have less transmission of data to the base station to lessening energy. Eliminate transmission of dreary data using meta-data courses of action as in SPIN protocol.

Improving clustering strategies for data aggregate to screen energy of the sensors. Improving In-Network amassing strategies to upgrade energy viability. In-Network accumulation means sending not entirely gathered values rather than rough qualities, in this way lessening power usage. Data disseminating is a method by which data and the request for the data are coordinated in the sensor network [81]. Data dispersal is a two stage process. In the first step, if a node is enthusiastic about a couple of events, like temperature or wetness, then it broadcasts its side interests to its neighbors infrequently and after that through the whole sensor network. In the second step, the nodes that have the requested data will send the data back to the source node in the wake of tolerating the requesting. . The standard refinement between data collection and data diffusing is, in data dispersal the entire Ad-hoc network including the base station can request the data while in data mixture all al the gathered data is at times transmitted to the base station. In addition, data complete data can be transmitted irregularly, while in data spread data is continually transmitted on hobby. Flooding is one 
indispensable protocol which fuses data spread strategy. Central investigation pack in data gathering is furnished towards sparing energy. Other investigation issues join upgrading security in data transmission and aggregate, dealing with tradeoffs in data combination i.e. tradeoffs between different focuses, for instance, energy use, latency and data accuracy, upgrading nature of organization of the data mixture protocols in regards to information transmission and end to end delay.

\subsection{Database Querying}

WSN's can potentially compass and screen a far reaching geographical reach making colossal measure of data. So sensor Networks should have the ability to recognize the inquiries for data and respond with the results. The data stream in a sensor database is by and large not the same as the data stream of the standard database due to the going hand in hand with diagram issues and necessities of a sensor network [62][63][65][66]: The Ad-hoc network are shaky since the nodes may get depleted and associations between diverse nodes may go down whenever of time yet data amassing should be impeded as pitiful as could be permitted. Sensor data is uncovered a bigger number of slips than in a protocol database due to impedance of signs and contraption racket. Sensor Networks produce data determinedly constantly and on a significant scale from the recognized miracle realizing need of updating the data a great part of the time; while a customary database is essentially of static and amassed in nature. Limited storage and uncommon of energy is another crucial constraint that needs to be managed in a sensor network database yet a standard database generally speaking contains a great deal of advantages and circle space is not an issue. The low level correspondence/communication primitives in the sensor Networks are arranged seeing named data rather than the node identifiers which are used as a piece of the standard Networks. Some investigation zones in sensor database fuse giving spatio-tamporal quering, multi-query streamlining, stockpiling plan, delineating a spread long term composed data stockpiling, should have low energy correspondence/communication overhead, diverse strategies for identifying with the sensor data, get ready and scattering inquiry parts, overseeing correspondence/communication dissatisfactions and arranging distinctive models for sending and managing a sensor database structures.

\subsection{The Sensor Networks Programming Models}

Quickly, programming designers are a considerable measure of concerned with low level purposes of enthusiasm like recognizing/sensing and node to node correspondence/communication raising a prerequisite for programming thoughts. There is far reaching examination development for illustrating programming models for sensor organizes in light of taking after issues [82]: Since the data assembled from the including marvel is not for all around helpful enlisting we oblige an open, event driven programming model. Resources in a sensor network are uncommon, where even an ordinary embedded OS eating up a few KB of considered too much. So programming models should help designers in making energy capable applications. We need to decrease the run time bumbles and flightiness since the applications in a sensor network need to run for a long compass without human intercession. Programming models should help engineers to form information exchange limit viable ventures and should be joined by runtime instruments that achieve transmission limit capability at whatever point possible. TinyOS with Nesc [12] and TinyGALS [83] are representations for this class. Upgrading programming straightforwardness in tongues, for instance, Nesc and galsC [84] itself gives enormous opportunities to research.

\subsection{Structural Design}

According to [76] nonattendance of a general sensor network development displaying is the basic component for at this moment compelling the progression in sensor Networks. Auxiliary arranging can be considered as a course of action of fundamentals and regulation for realizing a couple of functionalities nearby a game plan of interfaces, utilitarian sections, protocols and physical gear. Programming auxiliary designing is relied upon to overcome any block between unrefined hardware capacities and a complete system. The key issues that must be had a tendency to by the sensor auxiliary building are [77][78][79]: Several operations like constant seeing of the channel, encoding of data and trading of bits to the radio need to be performed in parallel. Also sensor events and data figurings must continue proceeding with while correspondence/communication is in headway.

A strong and versatile development displaying would allow component changes to be made for the topology with slightest redesign messages being transmitted. The system must be versatile to meet the broad assortment of target application circumstances since the WSN's to not have an adjusted game plan of correspondence/communication protocols that they must stick to. The basic arranging must give precise control over radio transmission timing. This need is driven by the necessity for ultra-low power correspondence/communication for data gathering application circumstances. The basic arranging must decouple the data way speed and the radio transmission rate in light of the fact that prompt coupling between get ready speed and correspondence/communication bit rates can provoke defective energy execution.

The [80] blueprint/design a novel SP reflection which progresses cooperation over the association and network layers to utilize compelled resources capably. A coupling together appearance in SP prompts supporting a blended sack of association layer headways and network protocols while taking care that doing thusly won't incite a critical loss of viability.

\subsection{Middleware}

A middleware for WSN should hold up headway, bolster, exploitation and execution of sensing-based applications. WSN middleware can be considered as an item base that glues together the network gear, working structures, network stacks and applications [86]. Diverse issues in arranging a middleware for WSN's are [87][88][89][90][91][92]: Middleware should give an interface to the diverse sorts of gear and Networks maintained by primitive working system reflections. Middleware should give new programming perfect model to give application specific API's instead of overseeing low level particulars. Efficient middleware game plans should hide the multifaceted nature included in masterminding individual nodes considering their capacities and gear development demonstrating. Middleware should fuse Networks to give consistent approaches by alterably acclimating to the changes in nature and giving solid data. Middleware should be flexible to the devices being altered 
depending upon the hardware limits and application needs. There should be straightforwardness in the middleware arrangement. Middleware is planned for giving a general structure however sensor Networks are themselves proposed to be application specific. Thus we need to have some tradeoff trying to clearing articulation and specificity. Sensor network middleware should support transportability, versatility and component network affiliation. Middleware arrangement should combine constant needs. Need of a message should be apportioned at runtime by the middleware and should be in light of the association. Middleware should support nature of organization considering various goals which are excellent to sensor Networks like energy, data, flexibility and gathering. Security has happened to principal noteworthiness with sensor Networks being passed on in mission separating zones like military, flight and in therapeutic field. A couple middleware structures have been planned to deal with the already expressed issues. Mate [94] is a middleware structural design for creating application specific virtual machines that executes on top of TinyOS. The design and execution of a middleware layer for totally understanding the capacity of remote sensor network is an open examination area which still needs to be investigated further. One need to diagram creator neighborly middleware building configuration which is insipid and should manage all the essential gear intricacies while serving to reduce the energy usage moreover give agreeable nature of sponsorship.

\subsection{Security}

Security in WSN's is as much an indispensable part as execution and low energy use in various applications. Security in a sensor network is greatly troublesome as WSN is being sent in combat area applications and also for surveillance, building watching, and burglar cautions and in segregating structures, for instance, air terminals and specialist's offices. Since sensor Networks are still a making development, researchers and specialists agree that their attempts should be amassed in making and organizing security from the beginning times of sensor applications change; in this way, they need to give a stronger and complete protection against unlawful activities and keep up robustness of the systems meanwhile. Taking after are the fundamental security necessities to which every WSN application should hold quick to [102][103][104][105][106][107][108]. Confidentiality is relied upon to ensure sensitive information is all around guaranteed and not revealed to unapproved pariahs. Mystery is required in sensor Networks to guarantee information going between the sensor Ad-hoc networks of the network or between the sensors and the base station; else it may achieve keeping an eye on the correspondence/communication. Authentication routines check the identity of the individuals in a correspondence/communication. In sensor Networks it is key for each sensor node point and the base station to have the capacity to affirm that the data got was genuinely sent by a trusted sender and not by a foe that bamboozled certifiable nodes into enduring false data. A false data can change the way a network could be foreseen. Lack of uprightness may realize mixed up information. Various sensor applications, for instance, sullying and human administrations checking rely on upon the genuineness of the information to limit; for e.g., it is inadmissible to have shameful information regarding the extent of the tainting that has happened. One of the various strikes dispatched against sensor Networks is the message answer strike where a foe may catch messages exchanged the node of nodes and answer them later to realize perplexity to the network. So sensor network should be expected for freshness; suggesting that the packets are not reused as needs be thwarting potential mix-up. In sensor Networks secure organization is needed at the base station level, since correspondence/communication in sensor network winds up at the base station. Issues like Key scattering to sensor nodes remembering the final objective to make encryption and coordinating information need secure organization. Furthermore, gathering systems require secure organization additionally, since each get-together of Ad-hoc network may consolidate endless that needs to be checked with each other and exchange data in a sheltered manner. Security and QoS are two opposite shafts in sensor Networks. Security parts like encryption should be lightweight so that the overhead is minimized and should not impact the execution of the network. Different sorts of perils in sensor Networks are Spoofing and adjusting the guiding information, uninvolved information gathering, node point subversion, sinkhole strikes, sybil ambushes, Denial of Service ambush and staying. The security issues acted by sensor Networks are a rich field for investigation issues. Arranging coordinating protocols having natural security highlights, another symmetric key cryptography for sensor Networks, illustrating secure data gathering protocols, plotting intrusion area structures and security systems for media sensors.

\subsection{QoS (Quality of Service)}

It is the level of service gave by the sensor Networks to its customers. [100] portray Quality of Service (QoS) for sensor orchestrates as the perfect number of sensors sending information towards information collecting sinks or a base station. Since sensor Networks are getting executed in more number of employments which fuses mission essential applications, for instance, military applications and nuclear plant checking applications; QoS is being given far reaching review as the events happening in these circumstances are of most compelling criticalness. The QoS coordinating computations for wired Networks can't be particularly associated with remote sensor orchestrates due to the going with reasons: The execution of the most wired coordinating computations relies on upon the openness of the precise state information while the dynamic method for sensor Networks make availability of accurate state information next to tremendous. Nodes in the sensor network may join, leave and rejoin and associations/links may be broken at whatever point. Therefore keeping up and re-securing the ways alterably which is a problem in WSN is not an enormous concern in wired Networks. Diverse QoS problems in sensor Networks are [95][96][97][98][99]: The QoS in WSN is troublesome in light of the way that the network topology may change dependably and the available state information for controlling is inherently free. Sensor Networks need to be supplied with the obliged measure of transmission limit so it has the limit accomplish a unimportant obliged QoS. Traffic is disproportionate in sensor network since the data is gathered from various nodes to a sink node point. QoS parts should be proposed for an uneven QoS forced development. Many a period directing in sensor Networks need to surrender energy viability to meet traffic necessities. In spite of the way that multihops diminish the measure of energy exhausted for data gathering the overhead joined without lifting a finger off the packet delivery. Moreover, abundance data makes routing a capricious task for data aggregate impacting thusly affecting QoS in WSN. Buffering in coordinating is gainful as it serves to get various packets before sending them. Yet, multihop 
controlling obliges buffering of huge measure of data. This imprisonment in bolster size will fabricate the deferral assortment that packets gain while going on unmistakable courses and even on the same course making it difficult to meet QoS necessities. QoS expected for WSN should have the ability to support adaptability. Counting or clearing of the Adhoc network should not impact the QoS of the WSN. One of the first protocol which had QoS hold up was the Sequential Assignment Routing (SAR) [101].

The zone of sensor network QoS by and large remains an unexplored examination district. Plotting a fitting QoS model, picking what number of layers need to be facilitated, support for heterogeneous Ad-hoc network, arranging QoS model for specific applications, sketching out QoS based protocols to organize them with other network like cell, LANs and IP, and laying out QoS through middleware layer.

\section{CONCLUSION}

WSN's have made far reaching mixture of defies that still entails to be had a tendency to. We have recognized an exhaustive once-over of dilemmas or research prospects associated with WSN's. Similarly it has been discussed some predominant protocols executing these problems mostly or by and large. The impact of remote sensor arranges on our ordinary life can be preferably appeared differently in relation to what ubiquitous Internet has bestowed to us. This field is doubtlessly going to issue us an immense prospect to revolutionize the way we see the globe in the present day.

\section{REFERENCES}

[1] "E.M.Royer et al", "A Review of Current Routing Protocols for Ad hoc Wireless Mobile Networks", IEEE Personal Communications, April 1999, pp 46-55.

[2] "I. Akylidiz et al", "A survey on sensor networks", IEEE Communications Magazine, Volume: 40 Issue: 8, August 2002, pp.102-114.

[3] "K. Akkaya et al", "A survey of Routing Protocols in Wireless Sensor Networks", Elsevier Ad Hoc Network Journal, 2005, pp 325-349.

[4] "Q. Jiang et al", "Routing Protocols for Sensor Networks", In proceedings of Consumer Communications and Networking Conference, 5-8 January, pp 93-98.

[5] "Ning Xu", "A survey of Sensor Network Applications".

[6] D.Culler, D.Estrin and M.Srivastava, "Overview of Sensor Networks", IEEE Computer Society, August 2004.

[7] http://www.tinyos.net

[8] "Levis, P et al", "The emergence of networking abstractions and techniques in tinyos", Proceedings of the First USENIX/ACM Symposium on Networked Systems Design and Implementation, NSDI 2004.

[9] "H.Abrach, et al, "MANTIS: System support for multimodal networks of in-situ sensors", In 2nd ACM International Workshop on Wireless sensor networks and Applications, pages 50-59, 2003.

[10] "S Park et al", "Embedded Sensor Networked Operating System", Proceedings of the Ninth IEEE International
Symposium on Object and Component-Oriented RealTime Distributed Computing, 2006., pp:117-124.

[11] Operating Systems, John Wiley Publication, 7th edition, 2006.

[12] "D.Gay et al", "The nesc language: A holistic approach to networked embedded systems", In Proceedings of the ACM SIGPLAN 2003 conference on Programming Language Design and Implementation, San Diego, California, USA.

[13] "A.Eswaran et al", "Nano-RK: An energy aware Resource Centric RTOS for sensor networks", In proceedings of the 26th IEEE International Real-Time Systems Symposium (RTSS'05) 2005, pp: 256-265.

[14] "M.Vieira et al", "Survey on Wireless sensor Network Devices", In proceedings of Emerging Technologies and Factory Automation, 2003 IEEE Conference, Volume: 1, 16-19, September 2003, pp: 537-544.

[15] http://computer.howstuffworks.com/mote1.htm

[16] "P.Zhang et al", "Hardware Design Experiences in ZebraNet", In proceedings of SenSys'04, November 3-5, 2004, Baltimore, USA.

[17] http://www.xbow.com

[18] "D.Puccinelli et al","Wireless Sensor Networks: Applications and Challenges of Ubiquitous Sensing", IEEE Circuits and Systems Magazine, Volume 5, Issue 3, 2005, pp: 19:31.

[19] "J.Hill et al","The Platforms Enabling Wireless Sensor Networks", Communications of the ACM, June 2004/Vol 47.No.6

[20] "G.Pottie et al", "Wireless Integrated Network Sensors", ACM Communications, Vol 43,No 5,PP 51-58,2000.

[21] "J.M.Kahn et al", "Wireless communications for smart dust", Electronic Research Laboratory, Vol.M98/2,1998.

[22] "A.Sinha et al", "Dynamic Voltage Scheduling using adaptive filtering of workload traces", In proceedings of the 11th International Conference on VLSI Design, 2001.

[23] "A.P Chandrakasan et al", "An Architecure for a PowerAware Distributed Microsensor Nodes", In IEEE workshop on Signal Processing Systems (SiPS'00), Lafayette, LA, Oct.2000.

[24] "Y.Zhou et al", "Overview of Power-Efficient MAC and Routing Protocols for Wireless Sensor Networks", Proceedings of the 2nd IEEE/ASME International Conference on Mechatronics and Embedded systems and applications, August 2006, pp 1-6.

[25] "P.P.Czapski", "A Survey: MAC Protocols for Applications of Wireless Sensor Networks", In proceedings of TENCON 2006, November 2006, Hongkong pp: 1-4.

[26] "T.Chiras et al", "Improved Medium Access Control for Wireless Sensor Networks - A study on the S-Mac Protocol", In proceedings of the 14th IEEE workshop on local and Metropolitan area networks, LANMAN 2005.

[27] "Wei Ye et al", "An Energy-Efficient MAC Protocol for Wireless Sensor Networks", IEEE Infocomm 2002. 
[28] "Ilker Demirkol et al", "MAC Protocols for Wireless Sensor Networks: A Survey", IEEE Communications Magazine, April 2006.

[29] "A. Warrier et al", "Mitigating Starvation in Wireless Sensor Networks", Military Communications Conference, 2006, MILCOm 2006, pp 1-5.

[30] "R.Lin et al", "Energy Efficient Medium Access Controls for Wireless Sensor Networks and Its State of Art”, IEEE 2004

[31] "K.Dantu et al", "RoboMote: Enabling mobility in sensor networks", In IEEE/ACM Fourth International Conference on Information Processing in Sensor Networks (IPSN/SPOTS) April 2005.

[32] "C. Wang et al", "A Survey of Transport Protocols for Wireless Sensor Networks", IEEE Network June 2006. Vol:20, Issue:3, pp:34-40.

[33] "V.S.Mansouri et al", "A Simple Transport Protocol for Wireless Sensor Networks", In proceedings of ISSNIP 2005.

[34] "Chien-Yih Wan et al", "Pump-Slowly, Fetch-Quickly (PSFQ): A Reliable Transport Protocol for Sensor Networks", IEEE Journal on selected areas in Communications, Vol 23, No 4, April 2005.

[35] "O.B.Akan et al", "Even To Sink Reliable Transport in Wireless Sensor Networks", IEEE/ACM Transactions on Networking, Vol 13, No 5, October 2005.

[36] "Limin Wang","Survey on Sensor Networks", Department of Computer Science Engineering, Michigan State University 2004.

[37] "D.Ganesan et al", "Networking Issues in Wireless Sensor Networks", Elsevier Science, 9th December 2005.

[38] "W.R.Heinzelman et al", "Energy-Efficient Communication Protocol for Wireless Microsensor Networks", IEEE Proc. Hawaii Int'l Conference. Jan 2000, pp 1-10.

[39] "P.Jiang et al", "A Study of Routing Protocols in Wireless Sensor Networks", In Proceedings of the 6th World Congress on Intelligent Control and Automation, June 21-23, 2006, Dalian, China.

[40] "R.Govindan et al", "Directed Diffusion: A scalable and robust communication paradigm for Sensor Networks", in Proceedings of the sixth Annual ACM/IEEE International Conference on Mobile Computing and Neworking(MOBICOM 2000), Boston MA, USA: ACM Press, August 2000, pp 56-67

[41] "Matthias Ringwald et al", "Deployment of Sensor Networks: Problems and Passive Inspection", In proceedings of Fifth International workshop on Intelligent solutions in embedded systems, Madrid, Spain 2007.

[42] "Ashar Ahmed et al", "Wired Vs Wireless Deployment Support for Wireless sensor Networks", TENCON 2006, IEEE region 10 conference, 1-3.
[43] "J.Li et al", "POWER: Planning and Deployment Platform for Wireless Sensor Networks", In proceedings of the Fifth International Conference on Grid and Cooperative Computing Workshops (GCCW'06), IEEE 2006.

[44] "Xia Zhenjie et al", "A Localization Scheme with Mobile Beacon for Wireless Sensor Networks", In Proceedings of International Conference on ITS Telecommunications Proceedings 2006.

[45] "R.Kumar Patro", "Localization in Wireless Sensor Networks with mobile beacons", 23rd IEEE Convention of Electrical and Electronics Engineers in Israel, 2004, pp 22-24.

[46] "A.Savvides et al", "Dynamic fine grained Localization in Ad hoc networks of sensors", In proc of Mobicom 2001, 166-179, July 2001

[47] "S. Pandy et al", "Localization of Sensor Networks considering energy accuracy tradeoffs", In proceedings of International conference on Collaborative computing: Networking, Applications and worksharing, 19-21 Dec 2005.

[48] "Lingxuan et al", "Localization for mobile sensor networks", In proceedings of the Tenth Annual International Conference on Mobile computing and Networking, Philadelphia 26th September-1 October 2004

[49] "Jeremy Elson et al","Wireless Sensor Networks: A New Regime for Time Synchronization", In proceedings of the First Workshop on Hop Topics in Networks 28-29 October 2002, Princeton, New Jersey, USA

[50] "S.PalChaudhuri et al","Adaptive Clock Synchronization in Sensor Networks", IPSN'04, April 26-27, Berkeley, California, USA

[51] "F.Sivrikaya et al","Time Synchronization in Sensor Networks: A Survey”, IEEE Network July/August 2004.

[52] "Bychkovskiy V et al","A Collaborative Approach to In place Sensor Calibration", 2nd International Workshop on Information Processing in Sensor Networks (IPSN'03), Polo Alto, April 2003, pages 301-316.

[53] "J.Feng et al","Model Based Calibration for Sensor Networks", Proceedings of IEEE Sensors, 22-24 october 2003, Vol: 2, pp:737-742.

[54] "K. Whitehouse et al","Calibration as Parameter Estimation in Sensor Networks",In Proceedings of WSNA'02 September 28, 2002, Atlanta,Georgia,USA, pp 59-67.

[55] "K.D.Wong","Physical Layer Considerations for Wireless Sensor Networks", IEEE International Conference in Network Sensing and Control, March 2004, pp 1201-06.

[56] "Tsung-Hsien Lin et al","Integrated low power communication system design for wireless sensor networks", IEEE Communications magazine 2004, pp 142-150. 
[57] "Tilak et al","A Taxonomy of Wireless Micro Sensor Network Models",Mobile Computing and Communication Review, Vol 6, No 2, 2002.

[58] "R.Rajagopalan et al","Data Aggregation Techniques in Sensor Networks: A Survey", IEEE Communications Surveys and Tutorials, 4th quarter, 2006.

[59] A.Boulis, Saurabh Ganeriwal, Mani B.Srivastava,"Aggregation in Sensor Networks: An energy-accuracy trade-off", Elseiver, Ad Hoc Networks 1 (2003) 317-331.

[60] "Xiaoxing Li","A survey on Data Aggregation in Wireless Sensor Networks", Project report for CMPT 765, Spring 2006.

[61] "E.J.Duarte-Melo et al", "Data gathering wireless sensor networks:Organization and Capacity", Elsevier, Computer Networks 43 (2003) 519-537.

[62] "Li Qin Zhuang et al","Data management for Wireless Sensor Networks:Research Issues and Challenges", In proceedings of 2005 International Conference on Control and Automation (ICCA2005), June 27-29, Budapest, Hungary.

[63] "Philippe Bonnet et al","Querying the physical world", IEEE Personal Communications, October 2000

[64] "R.Govendan et al","The sensor Network as a Database", Technical report 02-771, Computer Science Department, University of southern California, September 2002.

[65] "J.C.Navas et al","The Network is the Database:Data Management for Highly Distributed Systems", ACM SIGMOD 2001 May 21-24, Santa Barbara, California USA.

[66] "A.Deshpande et al","Model-Driven Data Acquisition in Sensor Networks", Proceedings of the 30th VLDB Conference, Toronto, Canada, 2004.

[67] "S.Ratnasamy et al","Data Centric Storage in Sensornets with GHT, A Geographic Hash Table", Mobile Network and Applications (MONET), Special Issue on Wireless Sensor Networks, Kluwer, mid 2003.

[68] "Bose P et al","'Routing with Guaranteed Delivery in Ad hoc Wireless Network", Wireless Networks, 7(6) (2001) 609-616, also in DealM'99, Seattle, August 1999, 48-45

[69] "C. Buragohain et al","Power Aware Routing for Sensor Databases", Proceedings of 24th annual joint conference of the IEEE Computer and communications societies, 1317 March 2005, Volume: 3, pp 1747-1757.

[70] "J.Gehrke et al","Query Processing in Sensor Networks", IEEE Pervasive computing, Vol. 03, no 1, pp. 46-55, JanMar 2004.

[71] "Yong Yo et al","Query Processing for Sensor Networks", Proceedings of the 2003 CIDR Conference.

[72] "S.R.Madden et al","TinyDB: An Acquisitional Query Processing System for Sensor Networks",ACM Transactions on Database Systems, Vol V, No N, 2004.

[73] "Philippe Bonnet et al","Querying the Physical World",

[74] http://telegraph.cs.berkeley.edu/tinydb
[75] http://cougar.cs.cornell.edu

[76] "D.Culler et al","Towards a sensor network architecture:Lowering the waistline",Proceedings of the hot topics of in Operating Systems (HotOS'05), International Computer Science Institute 2005.

[77] "System Architecture for Wireless Sensor Networks" by Jason Lester Hill, Ph.D dissertation, University of California at Berkeley.

[78] "S.Duan et al","Exploring Hierarchy Architecture for Wireless Sensor Network Management", IEEE 2006.

[79] "Jason Hill et al","System Architecture Directions for Networked Sensors", Proceedings of the ninth International conference on architectural support for programming languages and operating systems, pp: 93104, November 2000, Cambridge, Massachusetts, USA.

[80] "J.Polastre et al","A Unifying Link Abstraction for Wireless Sensor Networks", SenSys'05, November 2-4, 2005, San Diego, California, USA, ACM 2005.

[81] "Wensheng Zhang et al","Data Dissemination with Ring Based Index for Wireless Sensor Networks", IEEE Transactions on mobile computing, Vol 6, No 7, July 2007.

[82] "R.Sugihara et al","Programming Models for Sensor Networks: A Survey", ACM Transactions on sensor networks 2006

[83] "E.Choeng et al","TinyGALS: A programming model for event-driven embedded systems", in SAC'03, pp 698704.

[84] "E.Cheong et al","galsC:A Language for Event-Driven Embedded Systems", Proceedings of the Design, Automation and Test in Europe Conference and Exhibition (DATE'05), IEEE 2005.

[85] "U. Bischoff et al","A State based Programming Model and System for Wireless Sensor Networks", Proceedings of the fifth Annual IEEE International Conference on Pervasive Computing and Communicatins Workshops (PerComW'07).

[86] "S.Hadim et al","Middleware: Middleware Challenges and Approaches for Wireless Sensor Networks", IEEE Distributed Systems online 1541-4922, Published by the IEEE Computer Society, Vol 7, No 3, March 2006.

[87] "Karen et al","A survey of Middleware for Sensor Networks:State of the art and future directions", Proceedings of MidSens'06, November 27-December 1, 2006, Melbourne, Australia.

[88] "M.Wolenetz","Middleware Guidelines for Future Sensor Networks", First Annual International Conference on Broadband Networks, October 25-29, 2004, San Jose, California, USA.

[89] "K.Romer et al","Middleware Challenges for Wireless Sensor Networks", Mobile Computing and Communications Review, Volume 6, Number 2.

[90] "Yang Yu et al","Issues in Designing Middleware for Wireless Sensor Networks", IEEE Network, January/February, IEEE 2004. 
[91] "S. Hadim et al","Middleware for Wireless Sensor Networks", International conference on Parallel Processing Workshops, 2006, 14-18 August.

[92] "M.M.Molla et al","A survey of Middleware for Sensor Network and challenges", Proceedings of the 2006 International Conference on Parallel Processing Workshops (ICPPW'06), IEEE 2006.

[93] "Boulis et al",'Design and Implementation of a Framework for Programmable and Efficient Sensor Networks", In MobiSys 2003, San Francisco, USA, May 2003.

[94] "P.Levis et al","Mate: A Tiny Virtual Machine for Sensor Networks", In Proceedings of the 10th International Conference on Architectural Support for Programming Languages and Operating Systems, San Jose, CA, USA, October 2002.

[95] "D.Chen et al","QoS Support in Wireless Sensor Networks: A Survey", Proceedings of the 2004 International Conference on Wireless Network (ICWN 2004), Las Vegas, Nevada, USA, June 21-24, 2004

[96] "M.Younis et al","On Handling QoS traffic in Wireless Sensor Network", Procedings of the 37th Hawaii International Conference on System Science 2004.

[97] "James Kay et al","Quality of Service Analysis and Control for Wireless Sensor Networks", 2004 IEEE International Conference on Mobile Ad-hoc and Sensor Systems.

[98] "Yuanli Wang et al"," Requirements of Quality of Service in Wireless Sensor Networks", Proceedings of the International Conference on mobile communications and learning technologies (ICNICONSMCL'06) IEEE 2006.

[99] "Liudong Xing et al",'QoS Reliability of Hierarchical Clustered Wireless Sensor Networks", Proceedings of 25th IEEE International Conference on Performance, Computing and Communications, 10-12 April 2006

[100] "Ranjit Iyer et al",'QoS Control for Sensor Networks", IEEE 2003.

[101] 'K.Shhrabi et al",,'Protocols for self organization of a wireless sensor networks",IEEE Personal Communications, October 2000, pp 16-27.

[102] "Al-Sakib khan pathan et al","Security in wireless sensor networks: Issues and challenges", IEEE ICACT 2006.

[103] "Tanveer Zia et al","Security Issues in Wireless Sensor Networks", ICSNC, P.40, International Conference on Systems and Networks Communication (ICSNC'06), 2006.

[104] "Yong Wang et al","A survey of security issues in wireless sensor networks", IEEE Communications survey, 2nd quarter 2006, Volume 8, No 2.

[105] 'Djamel Djenouri et al","A survey of security issues in mobile ad hoc and sensor networks", IEEE Communications surveys, Fourth Quarter 2005, Volume 7 , No 4 .

[106] "Elaine Shi et al",'Designing Secure Sensor Networks",IEEE Wireless Communications, Volume 11, Issue 6, December 2004, pp: 38-43.

[107] "G.Guimaraes et al",'Evaluation of security mechanisms in wireless sensor networks", Proceedings of the 2005 Systems Communications (ICW'05)

[108] "J. Xiangyu et al","The security routing research for WSN in the application of intelligent transport system", Proceedings of the 206 IEEE International conference on mechatronics and automation, June 25-28, 2006, Luoyang, China. 